This is a post-peer-review, pre-copyedit version of an article published in Ann. Telecommun. 74, 697-715 (2019). The final authenticated version is available online at: https://doi.org/10.1007/s12243-019-00715-9

\title{
Dynamic Multicriteria Alternative Routing for Single- and Multi-service Reservation-Oriented Networks and Its Performance
}

\author{
Catarina Francisco 1 Lúcia Martin $\AA^{2}$ and Deep Medh ${ }^{3}$
}

\begin{abstract}
We propose a new Dynamic Multicriteria Alternative Routing (DMAR) method that applies to reservation-oriented networks. DMAR combines a dynamic alternative routing scheme with a periodic update of alternative paths according to a multicriteria algorithm that aims to balance the traffic between traffic flows in single service networks and also between services in multi-service environments. We conducted extensive simulations to compare the performance of DMAR with that of other reference alternative routing schemes in single and multi-service networks with several topologies and load scenarios, namely with non-sta-tionary traffic. We show that DMAR efficiently adjusts to traffic changes while often presenting better network performance than the reference alternative routing schemes, particularly in the multicriteria sense.
\end{abstract}

keywords: Multicriteria Optimization, QoS Routing, Dynamic Alternative Routing, Multi-service Networks, Blocking Probability, Implied Costs

\section{Introduction}

Reservation-oriented networks require a service request to meet a certain service guarantee before the request is admitted to the network. Most commonly known reservation-oriented networks are voice telephone circuit-switched networks and optical networks. Recent technologies such as multiprotocol label switching (MPLS) networks and software-defined networks (SDN) have the ability to provide reservation-oriented services that require service guarantees. While dynamic routing for reservation-oriented networks has received a lot of attention in the past three decades (starting with circuit-switched networks) to improve network performance, we found that certain aspects of dynamic routing in reservation-oriented networks have remained unexplored. For instance, how does the network perform when multiple criteria are considered? How about when multiple services are offered? How is the network traffic dynamism adapted by a dynamic routing scheme? Exploring this problem space spanning these three dimensions, we propose a dynamic multicriteria alternative routing (DMAR) scheme, along with its performance assessment.

\footnotetext{
${ }^{1}$ Nokia Solutions and Networks Portugal S.A., Estrada do Seminário 4, Edifício Conhecimento, Alfragide, 2610-171 Amadora, Portugal, catarina.francisco@nokia.com

${ }^{2}$ Electrical and Computers Engineering Department, University of Coimbra, 3030-290 Coimbra, Portugal, lucia@deec.uc.pt,

${ }^{3}$ Computer Science \& Electrical Engineering Department, School of Computing and Engineering, University of Missouri-Kansas City, Kansas City, MO 64110-2499 USA, dmedhi@umkc.edu
} 
More specifically, the contribution of our dynamic routing scheme is a combination of an event-dependent dynamic alternative routing scheme with a periodic update of alternative paths according to a multicriteria routing algorithm, in an attempt to simultaneously guarantee an efficient management of resources and a more equitable overall quality of service. The formulation of the routing problem for single service networks considers the minimization of the mean network blocking probability and of the maximal point-to-point blocking probability, and the formulation for multi-service networks aims at minimizing the bandwidth denial ratio (BDR) and the maximal service mean blocking probability. In this context, the purpose of the routing scheme proposed in this work is to periodically calculate a set of paths for the entire network that represent a compromise solution among the objective functions.

\subsection{Background and Related Work}

Dynamic alternative routing has been proposed as a way to improve network performance by allowing a second chance to a connection whose first choice path does not have the available resources to meet its QoS requirements. This routing strategy is mainly suited for metro and wide-area backbone networks with a fully or nearly-fully meshed topology where there is a set of admissible paths (instead of a single one) to be used.

Dynamic alternative routing has been widely studied and implemented in circuitswitched networks [1, 2, 3] as a way to improve network performance. It has also been more recently proposed in [4, 5] and suggested for several networking technologies such as ATM [6], optical networks [4, 7, 8, 9], IP networks [10] and MPLS networks [11, 12, 13]. The requirements for the use of dynamic routing in an MPLS environment are explained in detail in [13].

Dynamic Alternative Routing (DAR) [2] is an event-de-pendent scheme working as follows: a connection is offered to the first choice path (usually the direct-link path) and, if there are no available resources, the connection may overflow to a randomly chosen alternative path. If this alternative path fails, a new alternative path to be used in future incoming requests is randomly selected from within a set of admissible alternative paths, using the sticky random principle. DAR is particularly attractive because it is simple and based on local information, allowing the network to efficiently adjust to changes. For this reason, DAR was implemented by British Telecom (BT) and it has been proposed for several network technologies such as ATM [6], optical [7], IP [10] and MPLS [11, 12].

DAR was originally designed for fully meshed networks. Adaptive Alternative Routing (AAR) is presented in [7] as an adaptation of DAR to generic topology optical networks where the first choice path is the shortest path and a pool of variable size of alternative paths is tried in a set of different topologies. In this case, multiple alternative paths may be attempted by each call due to the use of the crankback feature. However, the sparser the network, the bigger the setup time that is experienced.

The approaches discussed so far are based on a single criterion such as to maximize carried traffic. However, in many situations, a selection of a path may need to be based on multiple criteria. The use of a multicriteria formulation enables to take advantage of the trade-offs between the objective functions explicitly involved in the problem in order to choose in each case appropriate non-dominated solutions. A non-dominated solution, or a Pareto optimal solution, is a feasible solution such that there is no other feasible solution which improves one objective function without worsening the value of any other objective. A state of the art review on multicriteria routing and network 
design models in telecommunications networks is presented in [14].

The single criterion behind the classical alternative routing methods such as DAR is the maximization of the carried traffic. However, it typically leads to a higher maximal point-to-point blocking probability value in a single service network model, and it does not guarantee equity in the quality of service provided to the various services in a multiservice model. An attempt to solve this problem is proposed with Multiple Objective Dynamic Routing method (MODR), defined for single and multi-service networks in [15] and [16], respectively. Work in [13] proposes a simplified version of MODR for multi-service networks, herein designated by Simplified MODR (SMODR), more suitable for a realistic network environment as the computational effort is very much reduced while good results can still be reached.

The main idea with MODR is to periodically calculate the "best" set of single alternative paths that represent a compromise solution among the objective functions. The formulation of the routing problem of MODR for single service networks considers two objectives: the minimization of the mean network blocking probability and the minimization of the maximal point-to-point blocking probability. On the other hand, for multi-service networks, a hierarchy with two optimization levels is considered: the high priority network level objective functions aim at maximizing the network expected revenue and minimizing the maximal service mean blocking probability; the less priority service level objective functions aim to minimize the service mean blocking probabilities and the maximal point-to-point blocking probability for each service. MODR is solved through a heuristic approach based on an exact bicriteria shortest path algorithm using blocking probabilities and implied costs [17, 15], where the implied costs measure the impact in terms of traffic loss due to a reduction in the available capacity upon the acceptance of a connection, and their minimization tends to an increase in the carried traffic.

In real networks where the traffic is intrinsically dynamic, a better network performance could be achieved with a scheme where the spreading of the overflow traffic (that could not be handled by the first choice path) across multiple alternative paths is allowed (as opposed to MODR with a single alternative path) to overcome situations of traffic unbalance. We posit that a set of potentially alternative paths periodically determined via a bicriteria algorithm according to the state of the network and in association with an event-dependent routing behavior would allow to better cope with diverse network topologies and changes in the traffic patterns while ensuring the fulfillment of QoS requirements, until a new set of paths is discovered in the following path update instant.

\subsection{Our contributions}

Our work proposes Dynamic Multicriteria Alternative Routing (DMAR), a routing scheme inspired by DAR and MODR. DMAR is based on a path caching mechanism and an event-dependent alternative routing strategy similar to DAR where path caching is periodically updated according to the state of the network by a bicriteria routing algorithm. The two metrics used for bicriteria are blocking probabilities and implied costs. As an adaptation to a generic topology, the first choice path is the shortest path such as in AAR. To properly validate if DMAR may represent an added value in real networks, the network performance of DMAR is evaluated in this work against the performance of DAR and MODR in a diversified simulation environment in terms of $i$ ) network model (single service and multi-service), $i i$ ) network topology (fully meshed and sparser) and iii) traffic matrices (with stationary and dynamic traffic). 


\section{The Dynamic Multicriteria Alternative Routing (DMAR) Method}

DMAR combines a centralized periodic state-dependent strategy like in MODR (and Dynamically Controlled Routing (DCR) [3]) with an event-dependent strategy like in DAR. As in MODR, a centralized routing processor periodically (every path update interval) estimates the offered traffic matrix for the following interval based on real-time measurements and performs, via a bicriteria routing algorithm based on fixed-point iterators, the computation of the new routing tables which are afterwards communicated to each originating node. The time it takes to process all these data is assumed as negligible when compared with the path update interval. Between path update instants, DMAR works similarly to DAR, and the alternative paths are locally updated on the basis of whether calls succeed or fail. DAR additionally employs trunk reservation as a first choice path protection mechanism while the heuristics of DMAR and MODR eliminate alternative paths. To assure the convergence of the fixed-point iterators in DMAR where multiple alternative paths are allowed, it was necessary to resort to heavy dampening techniques. Comparing with MODR, the heuristic of DMAR solves a problem with a larger solutions space being computationally more demanding.

\subsection{Single Service DMAR}

We first start by presenting our single service DMAR approach. Consider a single service network model $\left(S=\left\{S_{1}\right\}\right)$ where $N=\{1,2, \ldots,|N|\}$ is the nodes set. For a given time instant $t$, let $\overline{\mathscr{P} t}$ be the network routing plan which is constituted by the set of paths for the entire network, $a_{i j}^{t}$ be the offered load between nodes $i$ and $j$ and $B_{i j}^{t}$ be the average end-to-end blocking probability that is experienced by a connection being routed from node $i$ to node $j$. The computation of the blocking probabilities is based on a fixed-point iterator as in [15], extended to multiple alternative paths in [18], as a function of a particular routing plan, offered traffic matrix, network topology and links capacity (assuming Poissonian arrivals and statistical independence in the blocking of the links). The blocking probability on each link is calculated by applying the Erlang B formula. Additional details are described in [18]. The mean network blocking probability experienced by a connection in time instant $t$ is given by $B^{t}=$ $\sum_{i, j \in N} a_{i j}^{t} B_{i j}^{t} / \sum_{i, j \in N} a_{i j}^{t}$ and the formalization of the DMAR bicriteria routing problem for the single service case is:

(Problem $P_{1}$ )

$$
\begin{aligned}
& \frac{\min }{\mathscr{P} t} B=B^{t} \\
& \frac{\min }{\mathscr{P} t} B_{\max }=\max _{i, j \in N} B_{i j}^{t} \\
& \quad \text { s.t. equations of the traffic model enabling to calculate } \\
& \quad\left\{B_{i j}^{t}\right\} \text { in terms of }\left\{a_{i j}^{t}\right\} \text { and } \overline{\mathscr{P} t} \text { as described in [18]. }
\end{aligned}
$$

The purpose of DMAR in the single service network model is thus to periodically find the set of alternative paths that represent a good compromise solution in terms of the following network performance metrics: mean network blocking probability $B$ and maximal point-to-point blocking probability $B_{\max }$.

Discovering the "best" routing plan in the scope of DMAR can be computationally intensive. For the case of a fully meshed network, each pair of nodes can 
have $\sum_{i=0}^{N-2} C(N-2, i)$ possible sets of disjoint alternative paths, where $C(n, p)=$ $n ! /(p !(n-p) !)$ and $N$ is the number of nodes in the network. Considering that the solutions space includes the set of alternative paths for all $N *(N-1)$ node pairs, the solutions space for the single service routing problem includes $\left(\sum_{i=0}^{N-2} C(N-2, i)\right)^{N *(N-1)}$ different solutions. To cope with this complexity we use a heuristic based on an exact bicriteria shortest path algorithm.

The bicriteria shortest path strategy for DMAR uses two metrics for each link $l_{k}$ : i) the implied costs $m_{k}^{1}$, and ii) the blocking probabilities $m_{k}^{2}=-\log \left(1-B_{k}\right)$, where the $\log$ is used to transform the blocking probability in each link, $B_{k}$, into an additive metric. The computation of the implied costs is based on a fixed-point iterator as in [15], adapted by the authors in [18] to multiple alternative paths. Let $\mathscr{P}_{i j}$ be the set of paths between a given pair of end nodes $(i, j)$. For each path $p \in \mathscr{P}_{i j}$, consider that $\mathscr{L}_{p}$ denotes the set of links $l_{k}$ used by this path. The problem formulation for the bicriteria shortest path algorithm is as follows:

$$
\min _{p \in \mathscr{P}_{i j}} m^{u}=\sum_{l_{k} \in \mathscr{L}_{p}} m_{k}^{u}, \quad u=1,2 .
$$

Note that minimizing the path implied costs tends to minimize the mean network blocking probability while minimizing blocking in paths tends to minimize the maximal point-to-point blocking probability.

Consider further that $d$ is the bandwidth required by a connection on each link and that $n$ AltPaths is the maximum number of alternative paths allowed for pair $(i, j)$. The BSPA procedure is depicted in Algorithm 1 .

\footnotetext{
Algorithm 1 - BSPA Procedure (i, j, nAltPaths)

1. Compute the relative weights of $m^{1}$ and $m^{2}, \alpha_{1}$ and $\alpha_{2}: \alpha_{1} \times M 2=\alpha_{2} \times M 1 \wedge$ $\alpha_{1}+\alpha_{2}=1$, where $M 1$ and $M 2$ are the average values for each metric, respectively. These relative weights intend to give no preference to any of the metrics.

2. Calculate $\mathscr{P}_{i j}=\left\{p_{i j}^{1}, \ldots, p_{i j}^{M}\right\}$, where $\mathscr{P}_{i j}$ corresponds to the set of M two-arcs link disjoint alternative paths for pair $i \rightarrow j$ with an implied cost value lower than $d$.
}

3. Sort the paths in $\mathscr{P}_{i j}$ in ascending order of their euclidean distance to the ideal global optimal (which, in this case, is $(0,0)): \sqrt{\sum_{u=1}^{2}\left(\alpha_{u} \times m^{u}\right)^{2}}$.

4. If (M > nAltPaths) $\mathscr{P}_{i j}=\left\{p_{i j}^{1}, \ldots, p_{i j}^{n A l t P a t h s}\right\}$, where $\mathscr{P}_{i j}$ corresponds to the first nAltPaths paths in the previously ordered set.

5. $\mathscr{P}_{i j}$ is used as the new set of alternative paths for pair $i \rightarrow j$.

In each path update instant, the alternative paths for each pair of nodes in the single service network are determined according to the heuristic specified in Algorithm 2. Every time instant $t=\{n T: n=1,2, \ldots\}$, where $T$ is the path update interval, the two link metrics $\overline{m^{1}}$ and $\overline{m^{2}}$ are calculated considering the set of initial paths $\overline{\mathscr{P}}^{n}$ for the $n^{\text {th }}$ path update time instant and using a moving average traffic matrix estimate $\bar{A}^{n}$. By definition, any path whose implied cost is higher than the revenue that it generates should not be used. The revenue was considered equal to $d$ and, in the particular case 
of this work, it is assumed that each link has a capacity in units of bandwidth. A starting point for the heuristic of DMAR in order to achieve a good performance in terms of carried traffic is the sequential removal from the routing plan of the alternative path with the highest implied cost. In fact, the implied costs have already shown to be very effective in conducting to a good network performance in terms of carried traffic [17]. However, this is usually at the expenses of high maximal point-to-point blocking probability.

After the removal of the alternative paths with high implied cost, it is given priority (in terms of search for new alternative paths) to the pairs of end nodes responsible for the highest values of point-to-point blocking probabilities. A simple approach consists in sorting all pairs of end nodes $(i, j)$ in descending order of their point-to-point blocking probabilities $B_{i j}$. Considering $M_{i j}^{a}$ as the number of paths for pair $(i, j)$ in the set of paths of the current solution $\overline{\mathscr{P}}^{a}$, the search for a new set of alternative paths for that pair is done according to the bicriteria shortest path algorithm (BSPA), depicted in Algorithm 1 .

In BSPA the alternative paths to test in the new solution correspond to the first nAltPaths paths within the set of paths with an implied cost value lower than $d$ and sorted by ascending order of the euclidean distance to the ideal global optimum. This is an improved version of MMRA, the bicriteria shortest path algorithm in the MODR scheme. MMRA defines preference regions in the objective functions space, whose boundaries vary dynamically reflecting the network conditions, and the single alternative path to test in the new solution is the first solution to be found in the higher priority region, excluding the fixed first choice path.

Every newly discovered solution is compared against the "best"solution $\left(\overline{\mathscr{P}}^{*}\right)$ so far. The comparison criterion considers the best solution to be the one presenting the highest ratio of improvement in one objective, with the least degradation on the other. With this criterion and considering only the last solution found during the heuristic execution time, it is possible to reach a situation where the initial and final solutions are non-dominated, while a solution that dominates the initial solution has been found by the heuristic. For example, let's consider the following sequence of three tested solutions $\left(\mathrm{B}^{1}, \mathrm{~B}_{\max }^{1}\right)=(0.03,0.3),\left(\mathrm{B}^{2}, \mathrm{~B}_{\max }^{2}\right)=(0.032,0.27),\left(\mathrm{B}^{3}, \mathrm{~B}_{\max }^{3}\right)=(0.029,0.29)$. In this case, the final solution chosen by DMAR is the second one (while the third solution dominates the first solution). This limitation is acknowledged and accepted for the sake of simplicity.

\subsection{DMAR-S}

We now present DMAR as applicable to multi-service networks (DMAR-S). Consider a multi-service network model $\left(S=\left\{S_{1}, S_{2}, \ldots, S_{|S|}\right\}\right)$ where $N=\{1,2, \ldots,|N|\}$ is the nodes set. For a given time instant $t$, let $\overline{\mathscr{P} t}$ be the network routing plan, $a_{i j}^{t s}$ be the load offered by service $s$ between nodes $i$ and $j, d^{s}$ be the bandwidth required by a service $s$ connection on each link and $B_{i j}^{t s}$ be the average end-to-end blocking probability that is experienced by a service $s$ connection being routed from node $i$ to node $j$. The blocking probability on each link is calculated according to the methods in [19,20]. More details can be seen in [18]. Then, the mean network blocking probability experienced by a service $s$ connection in time instant $t$ is given by $B^{t s}=\sum_{i, j \in N} a_{i j}^{t s} B_{i j}^{t s} / \sum_{i, j \in N} a_{i j}^{t s}$ and the formalization of the DMAR bicriteria routing problem for the multi-service case is to consider minimization of bandwidth denial ratio $(B D R)$ and minimization of maximum service blocking: 

Algorithm 2 - DMAR
1. $\forall i, j \in N, \mathscr{P}_{i j}^{a}=\overline{\mathscr{P}}^{*}=\overline{\mathscr{P}}^{n}$
2. Calculate $\overline{m^{1}}, \overline{m^{2}}, \mathrm{~B}$ and $\mathrm{B}_{\max }$, for $\overline{\mathscr{P}}^{a}$ using the $\bar{A}^{n}$ estimate
3. $\min \mathrm{B} \leftarrow \mathrm{B}, \operatorname{minB}_{\max } \leftarrow \mathrm{B}_{\max }$, cycle $\leftarrow 0$, change $\leftarrow 0$
4. Identify $\mathscr{K}$, the set of pairs of nodes for which there is no two-links path with enough available bandwidth to carry all the blocked traffic in case of fixed routing (direct routing, for a fully meshed network)

5. While (cycle $<$ change +2 )

(a) $\max \operatorname{Cost} \leftarrow d$

(b) While ( $\max C o s t \geq d)$ do

(i) $\forall i, j \in N$, calculate $\max \operatorname{Cost}_{i j}=\max _{p^{m} \in \mathscr{P}_{i j}^{a} \backslash\left\{p^{1}\right\}} c_{p^{m}}$

(ii) Calculate maxCost $=\max _{i, j \in N} \max \operatorname{Cost}_{i j}$

(iii) If (maxCost $\geq d$ )

(A) Identify the pair $(i, j)$ which is responsible for the value of maxCost

(B) Calculate the new $\overline{\mathscr{P}}^{a}$ by eliminating from $\mathscr{P}_{i j}^{a}$ and $\mathscr{P}_{j i}^{a}$ the alternative path that is responsible for the value of maxCost

(C) Calculate $\overline{m^{1}}, \overline{m^{2}}, B$ and $B_{\max }$, for $\bar{A}^{n}$ and the new $\overline{\mathscr{P}}^{a}$

(D) ratio $_{B}=(\min B-B) / \min B$

(E) ratio $_{B_{\max }}=\left(\min B_{\max }-B_{\max }\right) / \min B_{\max }$

(F) If $\left(\right.$ ratio $_{B}+$ ratio $\left._{B_{\text {max }}}>0\right)$

- $\min B \leftarrow B, \min B_{\max } \leftarrow B_{\max }$

- change $\leftarrow$ cycle, $\overline{\mathscr{P}}^{*} \leftarrow \overline{\mathscr{P}}^{a}$

(c) Sort the pairs of nodes in descending order of $B_{i j}$, where $B_{i j}$ is calculated according to eq. 8 in [18]

(d) For $($ order $=1$; order $\leq N *(N-1) / 2$; order ++ )

(i) Identify the pair of nodes $(i, j)$ for which $B_{i j}$ has the order ${ }^{\text {th }}$ highest value

(ii) nAltPath $\leftarrow \mathrm{N}-1$

(iii) If $\left(B_{i j} \leq \mathrm{B} \wedge(i, j) \notin \mathscr{K}\right)$

- nAltPath $\leftarrow M_{i j}^{a}-1$

- If(nAltPath==0) nAltPath=1;

(iv) $\overline{\mathscr{P}}^{a}=\overline{\mathscr{P}}^{a} \backslash\left\{\mathscr{P}_{i j} \cup \mathscr{P}_{j i}\right\} \cup\left\{\mathscr{P}_{i j}^{B S P A(i, j, n A l t P a t h)} \cup \mathscr{P}_{j i}^{B S P A(j, i, n A l t P a t h)}\right\}$, where BSPA (Algorithm 11) determines the set of alternative paths for pair $(i, j)$ in the new solution

(v) Calculate $\overline{m^{1}}, \overline{m^{2}}$, B and $\mathrm{B}_{\max }$, for $\bar{A}^{n}$ and the new $\overline{\mathscr{P}}^{a}$

(vi) ratio $_{B}=(\mathrm{minB}-B) / \mathrm{minB}$

(vii) ratio $_{B_{\max }}=\left(\mathrm{minB}_{\max }-B_{\max }\right) / \mathrm{minB}_{\max }$

(viii) If $\left(\right.$ ratio $_{B}+$ ratio $\left._{B_{\max }}>0\right)$

- $\min \mathrm{B} \leftarrow \mathrm{B}, \operatorname{minB}_{\max } \leftarrow \mathrm{B}_{\max }$

- change $\leftarrow$ cycle, $\overline{\mathscr{P}}^{*} \leftarrow \overline{\mathscr{P}}^{a}$

(e) cycle $\leftarrow$ cycle +1

6. $\overline{\mathscr{P}}^{n+1} \leftarrow \overline{\mathscr{P}}^{*}$ 
(Problem $\left.P_{S}\right)$

$$
\begin{aligned}
& \min _{\overline{\mathscr{P} t}} B D R=\frac{\sum_{s \in S} \sum_{i, j \in N} a_{i j}^{t s} B_{i j}^{t s} d^{s}}{\sum_{s \in S} \sum_{i, j \in N} a_{i j}^{t s} d^{s}} \\
& \min _{\overline{\mathscr{P} t}} \max B=\max _{s \in S} B^{t s} \\
& \quad \text { s.t. equations of the traffic model enabling to calculate } \\
& \quad\left\{B_{i j}^{t s}\right\} \text { in terms of }\left\{a_{i j}^{t s}\right\} \text { and } \overline{\mathscr{P} t} \text { as described in [18]. }
\end{aligned}
$$

Note that the typical single objective in classical alternative routing methods is the maximization of the carried traffic that does not guarantee the fairest traffic management among different services, measured by the maximal service mean blocking probability. The purpose of DMAR in the multi-service network model is thus to find a good compromise solution in terms of the mean bandwidth denial ratio $B D R$ [11] and the maximal service mean blocking probability $\max B$. Note that the calculation of $B D R$ takes into account the effect of having services with different bandwidths all attempting to access the same network resources.

We now emphasize the difference in terms of routing problem formulation between DMAR and MODR for the multi-service case. While DMAR attempts to find good compromise solutions between $B D R$ and $\max B$, the formulation of MODR considers a hierarchy with two optimization levels: the more priority network level objective is the same as in DMAR; the less priority service level objective functions aim to minimize the service mean blocking probabilities and the maximal point-to-point blocking probability for each service.

The heuristic for DMAR-S is presented in Algorithm 3 and it results from the extension of Algorithm 2 to a multi-service network, through the replacement of $B$ by $B D R$ and $B_{\max }$ by $\max B$.

Algorithm 3 - DMAR-S

1. steps 144 in Algorithm 2, considering all services

2. While (cycle $<$ change +2$)$

(a) For ( $=1 ; \mathrm{s} \leq|S|$; $\mathrm{s}++) / /$ runs through all services $s \in S$

i. steps 5(a) - 5(e) in Algorithm 2 for service s

3. step 6in Algorithm 2

\section{Performance Evaluation}

We now report on conducting studies on performance of DMAR against the performance of DAR and MODR in a biobjective sense to validate whether the use of DMAR may represent an added value. Both DMAR and DAR allow a set of alternative paths to be used for each pair of nodes. However, while DAR has a fixed set of alternative paths and it applies a trunk reservation mechanism in each link $(\sqrt{\text { capacity }} / 2)$ [7] to deal with congestion situations, DMAR periodically calculates through a bicriteria routing algorithm and according to the state of the network the alternative paths to use 
in the following time interval. On the other hand, the comparison between MODR and DMAR sheds light into performance differences concerning the potential use of a single or multiple alternative paths to share the overflow traffic for each pair of nodes.

This performance study is done in diversified scenarios namely in different network models (single service and multi-service) and topologies (fully meshed and sparser networks) in several load situations, with both stationary and dynamic traffic patterns, which were obtained based on a voice service traffic pattern made available for a 24 hours period, courtesy of Sprint [21]. The traffic patterns used in this study are described later in Appendix $\mathrm{A}$ and they are used for five test network scenarios (derived from a 10-node network) as described in Appendix B, where Networks A, B and C are used for studying the single-service case, while Networks D and E are used for studying the multi-service case.

Recall that alternative routing is often studied and applied to fully meshed networks, where the first choice path is the direct link and there are $N-2$ alternative paths. However, in sparse topologies, the first choice path may not necessarily be the direct link for every node pair, the number of alternative paths may not be $N-2$ and the maximum allowed size for the admissible paths may be difficult to establish. Thus, we evaluated DMAR in both these two types of topologies.

It is also important to analyze the performance dependency of the routing methods for different traffic matrices. Global congestion situations evaluate if the routing methods are capable of avoiding the network performance degradation due to the excessive use of alternative (usually longer) paths. On the other hand, a dynamic traffic pattern is the most effective way to test a dynamic routing strategy as it represents a more realistic network environment.

We used an existing discrete-event connection simulator [22, 23], written using the CSIM package [24]. By extending this simulator, we implemented our scheme to evaluate its network performance. This simulator provides generators of stationary and dynamic traffic, namely for sinusoidal behavior in the latter case, and it allows the definition of a multi-service environment. The following is assumed: all traffic flows are homogeneous Poissonian and independent and service times are negative exponentially distributed. For each simulation scenario, 5 independent simulation runs were performed.

A typical value in circuit-switched networks for the path update interval is 10 seconds. However, to reduce the computational effort in this study, DMAR and MODR periodically calculate, every 1 minute, the routing plan to be used in the next time interval according to an estimated offered traffic matrix. In the simulator, the estimated offered traffic $\tilde{x}$ in the $n^{\text {th }}$ time interval for traffic flow $f$ is obtained from an estimate $\tilde{X}_{f}(n-1)$ of the offered traffic in the previous interval calculated from on-line measurements, for the same traffic flow, by using a first order moving average iteration: $\tilde{x}_{f}(n)=(1-b) \tilde{x}_{f}(n-1)+b \tilde{X}_{f}(n-1)$ with $b=0.9$.

\subsection{Single service network model}

Single service test networks were engineered with a single service with the required bandwidth $d=1$ and an average call duration of 1 minute, representative of voice.

\subsubsection{Stationary Traffic Matrices}

Often in the literature, a dynamic routing scheme has been tested by generating stationary traffic although this does not represent a realistic situation; we also do so for 


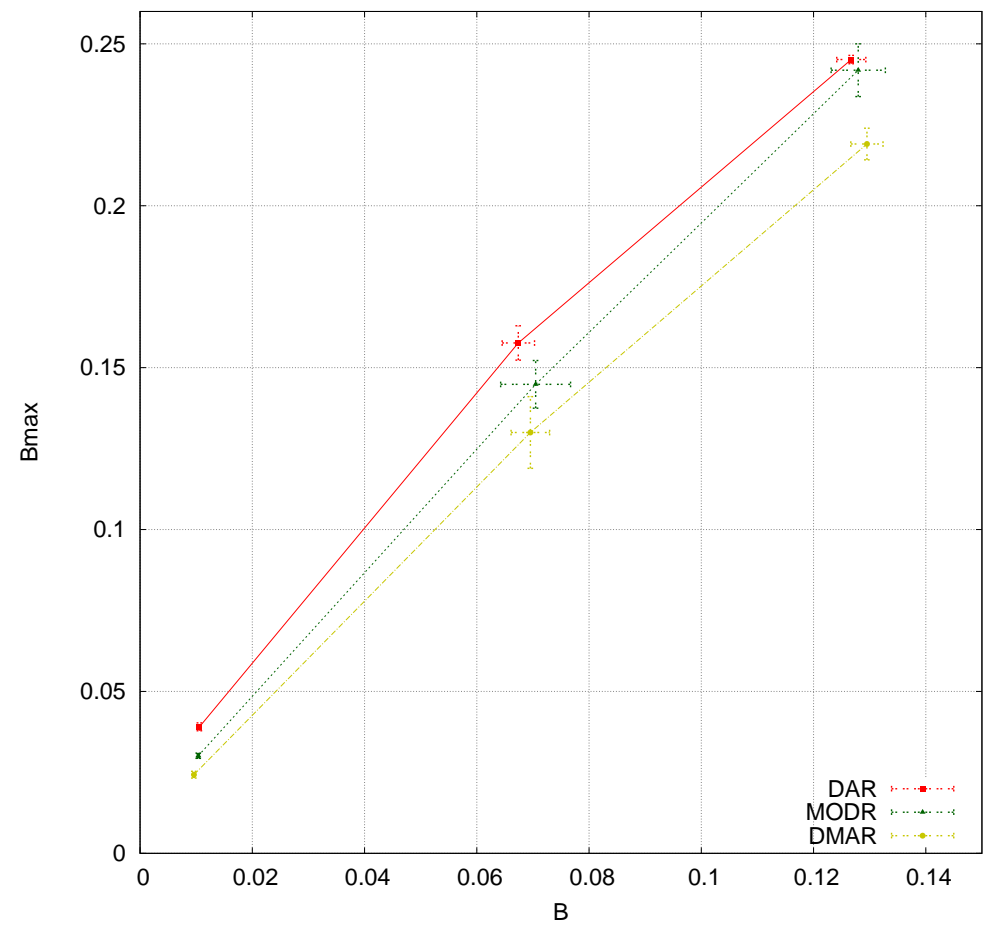

Figure 1: Network A - Nominal load and global congestion (10\% and 20\%).

the baseline study. Results for more realistic dynamic traffic are presented later in Section 3.1.2.

We begin with the comparative performance of DMAR, DAR and MODR in two 10-node networks (Network A and B) with different topologies (fully meshed and sparser, respectively) presented in Table 2 in Appendix B, and for a set of stationary traffic matrices inspired by a voice service traffic pattern for one of the busiest hours in [21] (see "BH" traffic in Table 1 in Appendix A.1). The reference traffic matrix is herein designated by the nominal load situation and its performance is compared against $10 \%$ and $20 \%$ global congestion situations in Figures 1 and 2 for Networks A and $\mathrm{B}$, respectively.

We represent the bicriteria nature of DMAR and MODR methods using Cartesian graphs where the axis correspond to each of the objective functions considered. The simulation results in Figures 1 and 2 for the network performance, measured in terms of $B$ and $B_{\max }$, as well as all the following simulation results are the midpoints of the confidence intervals calculated with a $95 \%$ confidence level and obtained using the method of independent replications.

We observe that for the nominal load situation, DMAR performs better (lower B and $\mathrm{B}_{\max }$ ) than DAR and MODR in both fully meshed (A) and sparser (B) networks. In regard to $10 \%$ and $20 \%$ overloaded situations, we observe that the values of $B_{\max }$ obtained with DMAR are significantly lower than the ones obtained with DAR and the $B$ values of DMAR are slightly higher than the corresponding values of DAR. However, the average B values of DMAR are within the $95 \%$ confidence interval of the B values for DAR; therefore, they are comparable. It may be noted that MODR was not found to be the method with the best value either for $\mathrm{B}$, or $\mathrm{B}_{\max }$. 


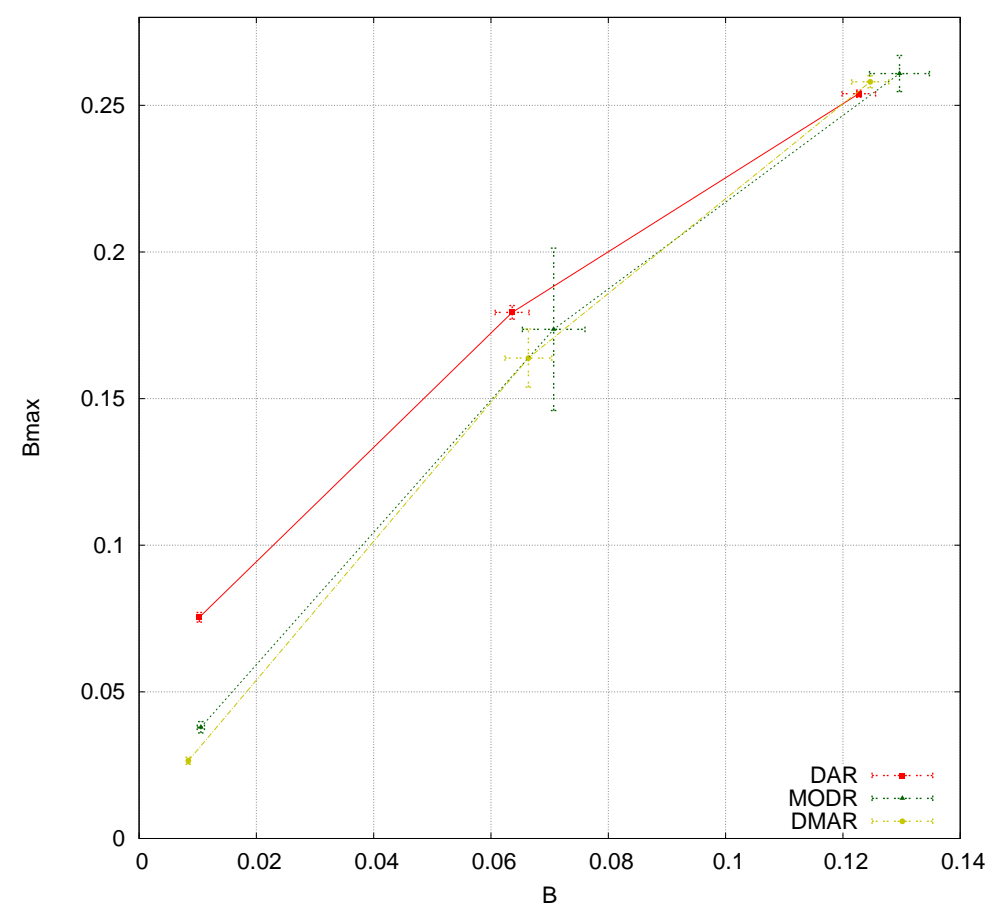

Figure 2: Network B - Nominal load and global congestion (10\% and 20\%).

\subsubsection{Dynamic Traffic Matrices}

To understand how traffic fluctuations affect the routing methods performance we now present results for dynamic traffic matrices, following the sinusoidal-based dynamic traffic model described in Appendix A.1. Figure 3 shows the offered traffic curves for a scenario where the traffic offered by each node to any other node follows a sinusoidal waveform with the average (Avg) equal to the "BH" value with a peak amplitude (A) of $5 \%$ of that value, and wave periods $(\mathrm{T})$ of 117,101 and 128 minutes, sequentially assigned according to the ordered list of node pairs. Figure 4 shows the total offered traffic curve for the $5 \%$ and $10 \%$ peak amplitude variation cases.

Because the traffic pattern varies over time, the results presented next correspond to 24-hours simulations, where the first 4 hours correspond to a warmup period (which is discarded from the analysis). Consequently, the performance results in Figures 5 |6 and 7.8 for Networks A and B, respectively, correspond to the ]4,24]h=]14400,86400]s period. Each 15 minutes interval $B$ and $B_{\max }$ are calculated and the curves in figures result from linear interpolation between the mean values of $B$ and $B_{\max }$, respectively, for 5 simulation runs. Note also in the legends the identification of the pair of nodes responsible for the value of $\mathrm{B}_{\max }$ for each of the methods. We observe that the performance of DMAR is the best for Network A in both 5\% and 10\% peak amplitude situations. For Network B, in both situations, the curve of B tends to be slightly better for DAR in the peak values at the expense of much worse $B_{\max }$ values.

To test the routing methods in a more realistic and challenging environment, the " 7 BHs" traffic scenario in Appendix A.1 considers the mapping into sinusoidal waves of the Sprint offered traffic matrices for the seven busiest hours period (] $10,17] \mathrm{h})$. Figure 9 shows the offered traffic curves for this scenario. The total offered traffic and the traffic 


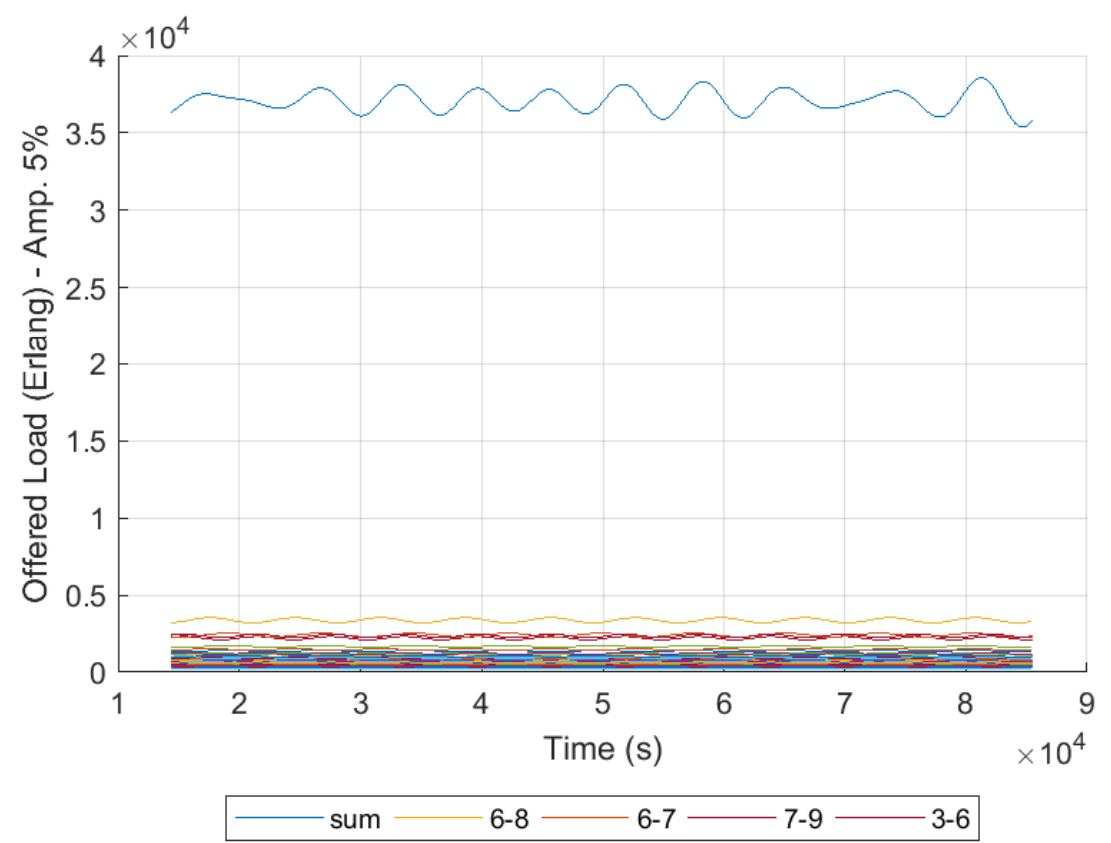

Figure 3: Offered traffic curves (per pair of nodes and total value) for the dynamic traffic with $\mathrm{A}=5 \%$.

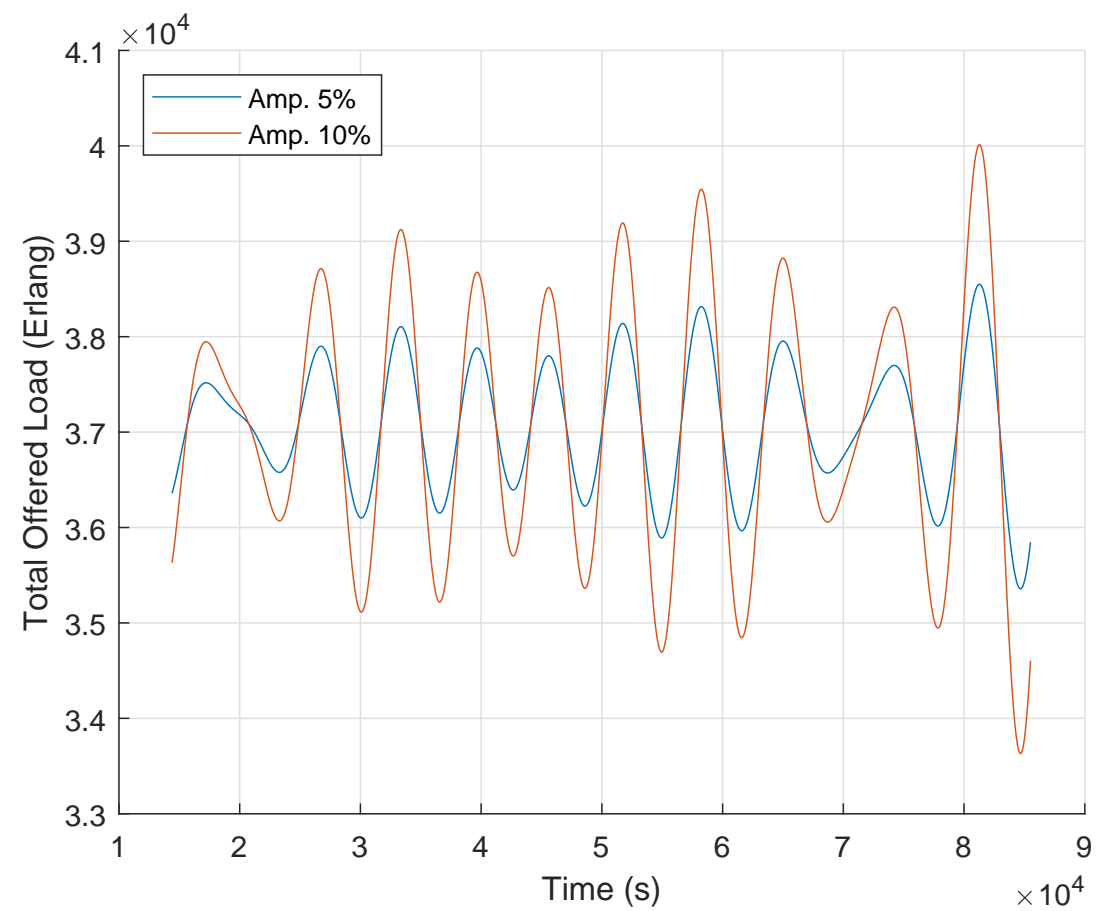

Figure 4: Offered traffic curves for the dynamic traffic with $\mathrm{A}=5 \%$ and $\mathrm{A}=10 \%$. 

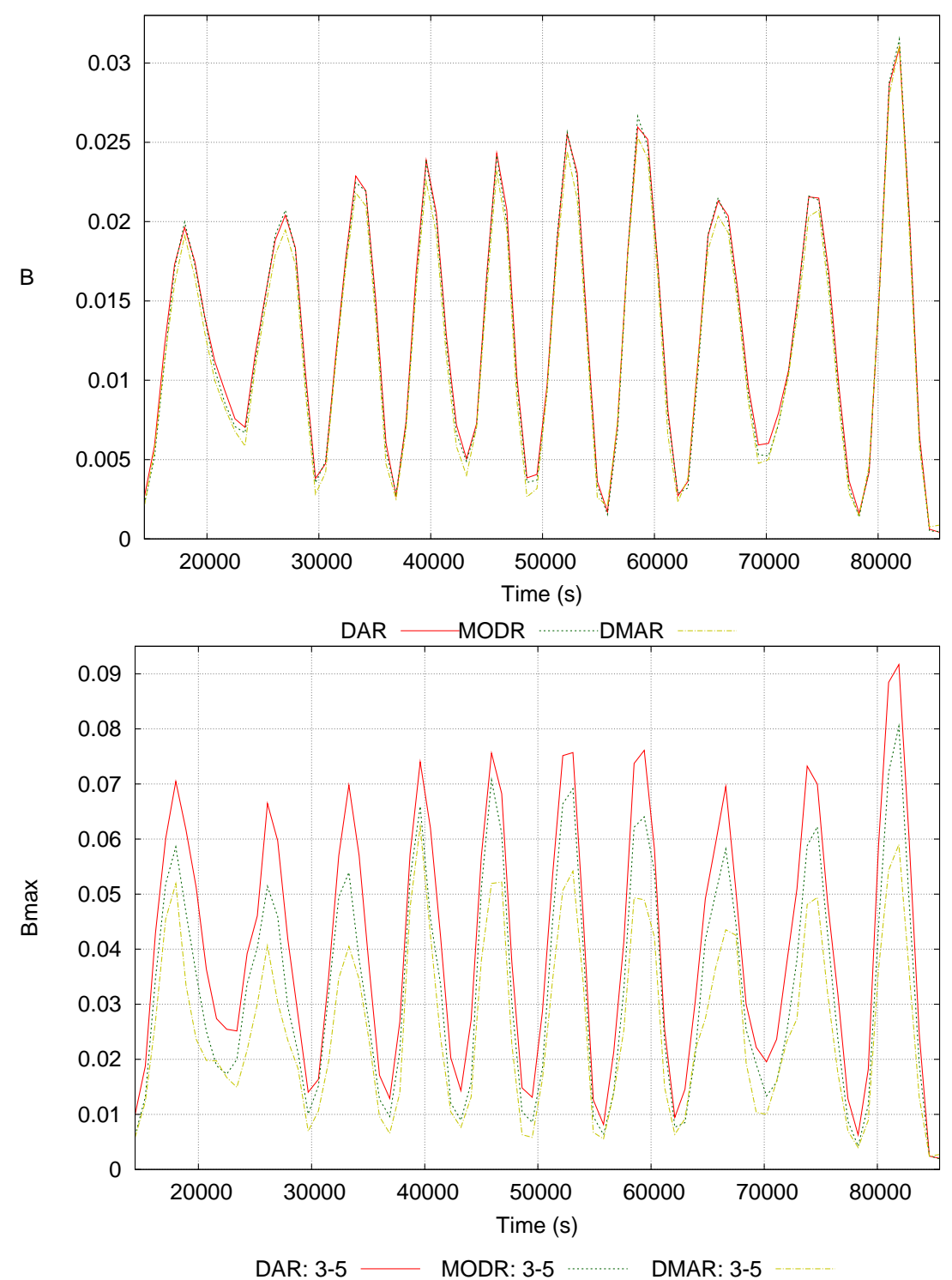

Figure 5: Network A - dynamic traffic (Avg="BH"; $A=5 \%$; $(\min )=101,117$ and 128). 

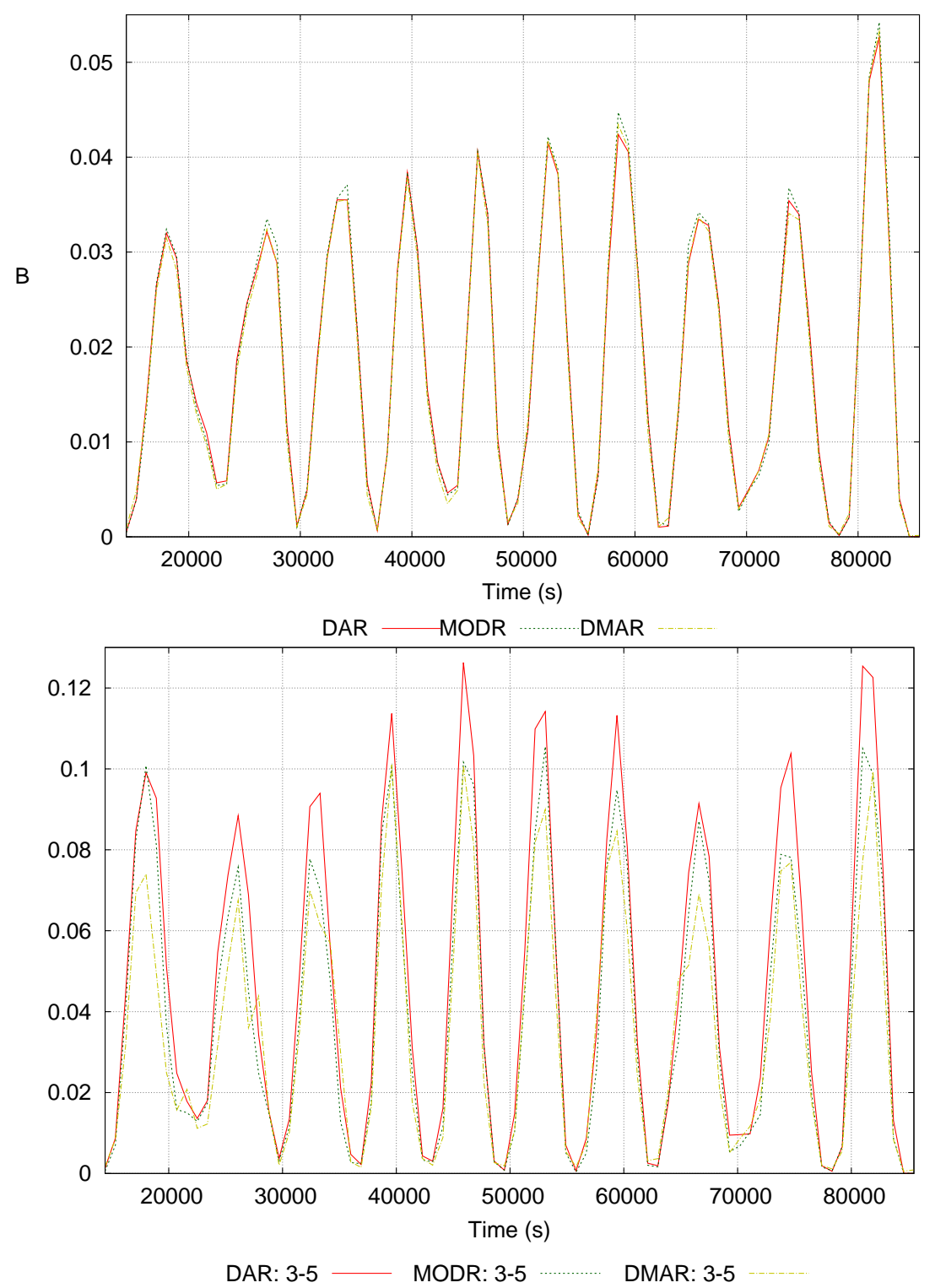

Figure 6: Network A - dynamic traffic (Avg="BH"; $\mathrm{A}=10 \% ; \mathrm{T}(\min )=101,117$ and 128). 

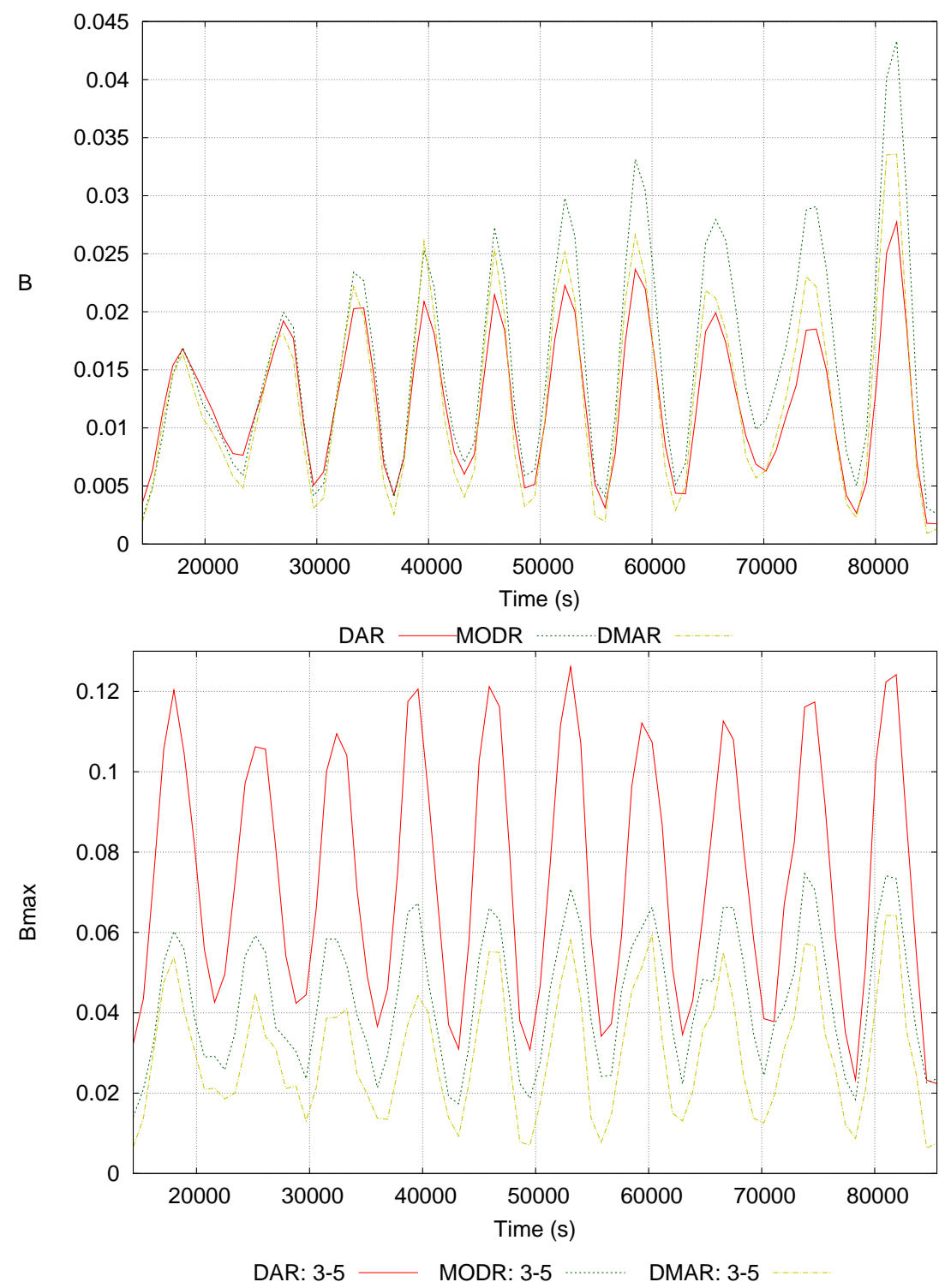

Figure 7: Network B - dynamic traffic (Avg="BH"; $A=5 \% ; \mathrm{T}(\min )=101,117$ and 128). 

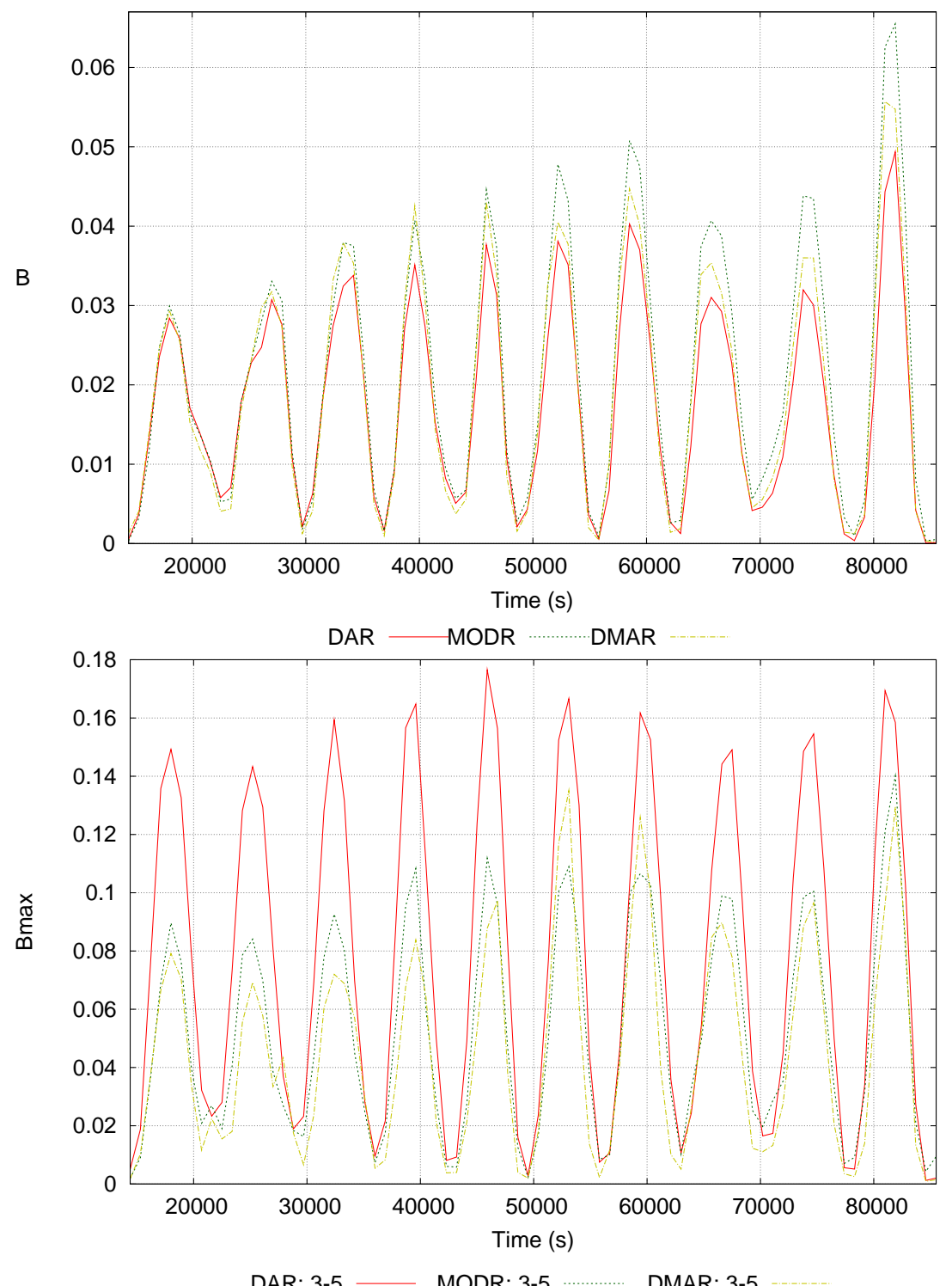

Figure 8: Network B - dynamic traffic (Avg="BH"; $\mathrm{A}=10 \%$; $\mathrm{T}(\min )=101,117$ and 128). 


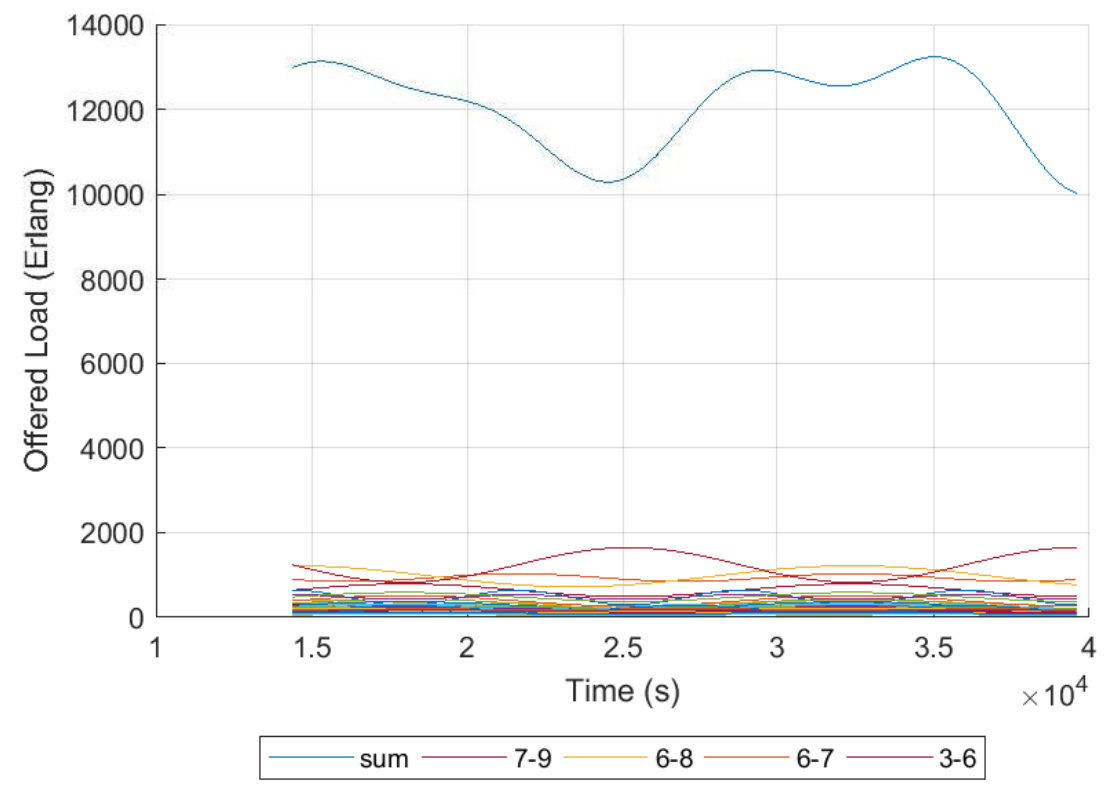

Figure 9: Offered traffic curves (per pair of nodes and total value) for the "7 BHs" traffic.

corresponding to the more demanding pairs of nodes are highlighted in the legend. This traffic was tested in Network C (see Table 2 in Appendix B). The performance results in Figure 10 indicate that DMAR presents the best performance for both metrics.

Note also in Figure 10 the much worse comparative performance of $\mathrm{B}$ curve for the MODR method in some time intervals ([14400,16200]s, [21600,28800]s and [36900,38700]s). The reason is that MODR only allows a single alternative path for each pair of nodes and there is no single alternative path capable of carrying the overflow traffic of pair 7-9 (the pair responsible for the $B_{\max }$ curve and that in average offers more traffic to the network) in the mentioned intervals. In fact, the worse performance occurs in the time intervals corresponding to the peaks of offered load to pair 7-9.

To test this assumption, a modification to MODR (herein designated as MODR+) was considered: in the beginning of each path update instant, the links whose occupation is higher than a threshold (99\%) are marked as being congested and cannot be used for alternative routing.

MODR+ was tested with a short path update interval (10 seconds) [25]. It is possible to conclude that the limitation of MODR was identified because the performance curve of B for MODR+ is more similar to the ones of the remaining routing methods. This evidence further supports DMAR and the use of multiple alternative paths to share overflow traffic. Note that, for consistency purposes the performance results for MODR in Figure 10 concern a 1 minute path update interval. However, no significant difference in terms of performance is identified between MODR with a 10 seconds and a 1 minute path update interval.

\subsection{Multi-service network model}

The multi-service networks in this study were engineered with three services with the required bandwidth $d=[1,6,10]$ and call duration $h=[1,5,10]$ minutes for services $S_{1}$, 

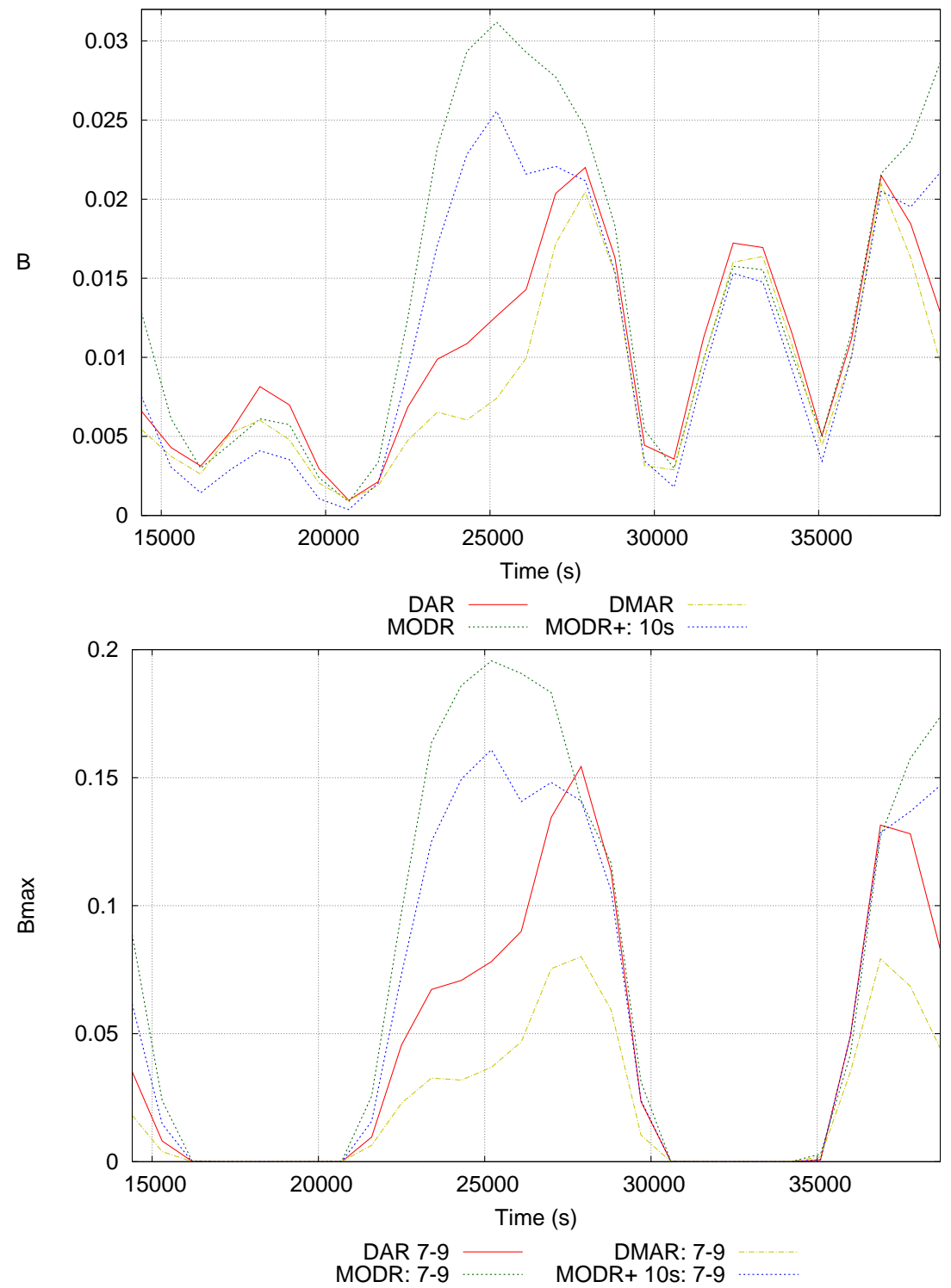

Figure 10: Network C - dynamic traffic (“7 BHs”). 
$S_{2}$ and $S_{3}$, respectively. Similarly to the approach followed in the single service network model, this simulation study includes several load situations, with both stationary and dynamic traffic.

For the multi-service network model a simplified version of MODR (herein designated by Simplified MODR or SMODR) is used instead. SMODR was firstly proposed in [13] and it is more adaptive than MODR to realistic multi-service network environments due to its reduced computational effort.

\subsubsection{Stationary Traffic Matrices}

The multi-service study begins by analyzing the performance of DMAR, DAR and SMODR for Network D (see Table 2 in Appendix B for a set of stationary traffic matrices, namely for nominal load ("BH" traffic extended to multi-service, as defined in Appendix A.2) as well as for $10 \%$ and $20 \%$ global congestion. Figure 11 shows that DMAR presents the best performance (lower $B D R$ and $\max B$ values) in all the tested scenarios. We observe that DMAR allows greater fairness among the different services (smaller discrepancies in terms of the service mean blocking probability values for each of the services) while DAR benefits services $S_{1}$ and $S_{2}$ with a much lower service mean blocking probability in detriment of a much higher value for service $S_{3}$. In fact, the mean blocking probability of service $S_{1}$ for DAR is so low in any of the load situations that its value cannot be observed in the presented bar chart.

\subsubsection{Dynamic Traffic Matrices}

Multi-service networks present an increased challenge, specially in situations where the traffic pattern varies in time. Figure 12 shows the performance results in Network E (see Table 2 in Appendix B) for a traffic pattern based on the one exhibited by the single service Sprint network during the seven busiest hours period, herein expanded to multi-service, as described in A.2 Note in the legend the identification of the service that is responsible for the value of $\max B$ for each of the methods.

We observe that DMAR performs better than SMODR. Furthermore, DMAR generally presents the best performance in terms of a bicriteria approach when comparing to DAR. Note that the biggest challenge when comparing with the performance of DAR lies near $t=15000 \mathrm{~s}, t=30000 \mathrm{~s}$ and $t=35000 \mathrm{~s}$, which correspond to the peaks of the total offered traffic curve where the accuracy of the offered traffic estimates is more critical. As already observed for the stationary traffic, Figure 12 also shows that DMAR is the method guaranteeing greater fairness among the different services for the dynamic traffic.

\subsection{Summary of Key Observations}

Based on our comprehensive studies presented above, we now summarize the key observations:

- For single service networks with stationary traffic, DMAR performs better than both MODR and DAR for nominal load, while they are comparable in $10 \%$ to $20 \%$ overloaded situations.

- For single service networks with dynamic traffic, DMAR has better performance for fully-mesh networks, while DMAR and DAR are comparable (DMAR 

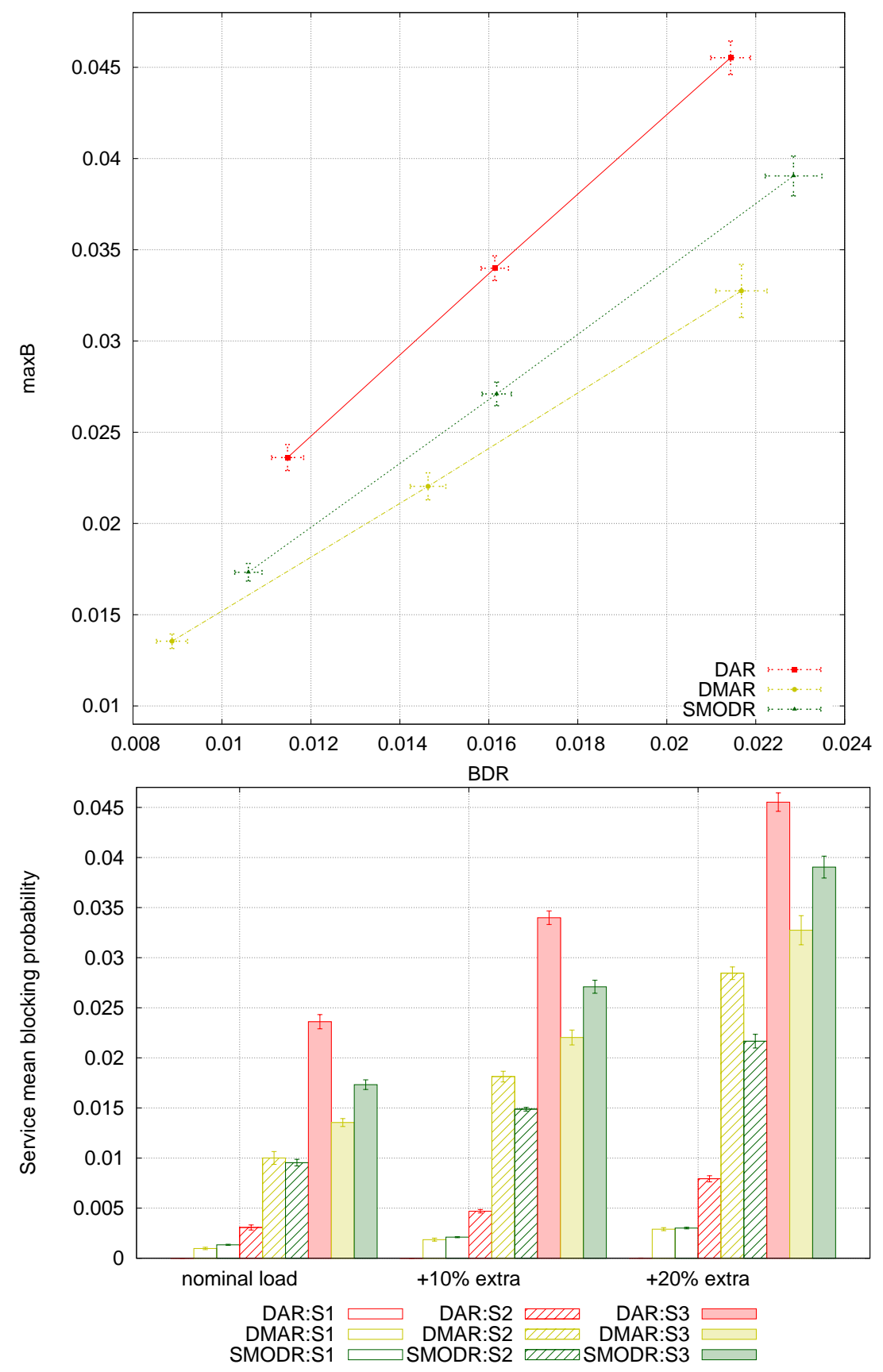

Figure 11: Network D - Nominal load and global congestion (10\% and 20\%). 
presents higher B and lower Bmax than DAR) for nearly fully-mesh networks in certain situations.

- For multi-service networks with stationary traffic, DMAR is found to perform better than the other schemes and is also more fair.

- For multi-service networks with dynamic traffic, DMAR performs better than the other schemes.

\section{Conclusion}

In this work, we consider reservation-oriented networks where dynamic alternative routing schemes are employed. In particular, we propose DMAR, a new dynamic alternative routing method with a multicriteria formulation. We first present DMAR for single service and then expanded it for multi-service networks. Our scheme is applicable to generic topologies. We evaluated DMAR against other reference routing schemes in a diversified simulation environment including several load scenarios like global congestion situations and dynamic traffic patterns. The obtained results show that DMAR efficiently adjusts to network changes while ensuring the satisfaction of QoS requirements.

Acknowledgements: Lúcia Martins has been supported by FCT (Fundação para a Ciência e a Tecnologia) under project grant UID/MULTI/-00308/2013 and by FEDER Funds and National Funds through FCT under the project CENTRO-01-0145-FEDER029312.

\section{References}

[1] Ash, G.: Dynamic Network Evolution, with Examples from AT\&T's Evolving Dynamic Network. IEEE Communications Magazine pp. 26-39 (1995)

[2] Gibbens, R.: Dynamic routing in circuit-switched networks: The dynamic alternative routing strategy. Ph.D. thesis, University of Cambridge (1988)

[3] Girard, A.: Routing and Dimensioning in Circuit-Switched Networks. AddisonWesley Publishing Company (1990)

[4] Wang, M., Li, S., Wong, E., Zukerman, M.: Blocking Probability Analysis of Circuit-Switched Networks With Long-Lived and Short-Lived Connections. J. Opt. Commun. Netw. 5(6) (2013)

[5] Wang, M., Li, S., Wong, E., Zukerman, M.: Performance analysis of circuit switched multi-service multi-rate networks with alternative routing. IEEE/OSA Journal of Lightwave Technology 32(2), 179-200 (2014)

[6] Conte, M.: Dynamic Routing in Broadband Networks. Kluwer Academic Publishers (2003)

[7] Katib, I., Medhi, D.: Adaptive Alternate Routing in WDM networks and its performance tradeoffs in the presence of wavelength converters. Optical Switching and Networking 6(3), 181-193 (2009) 

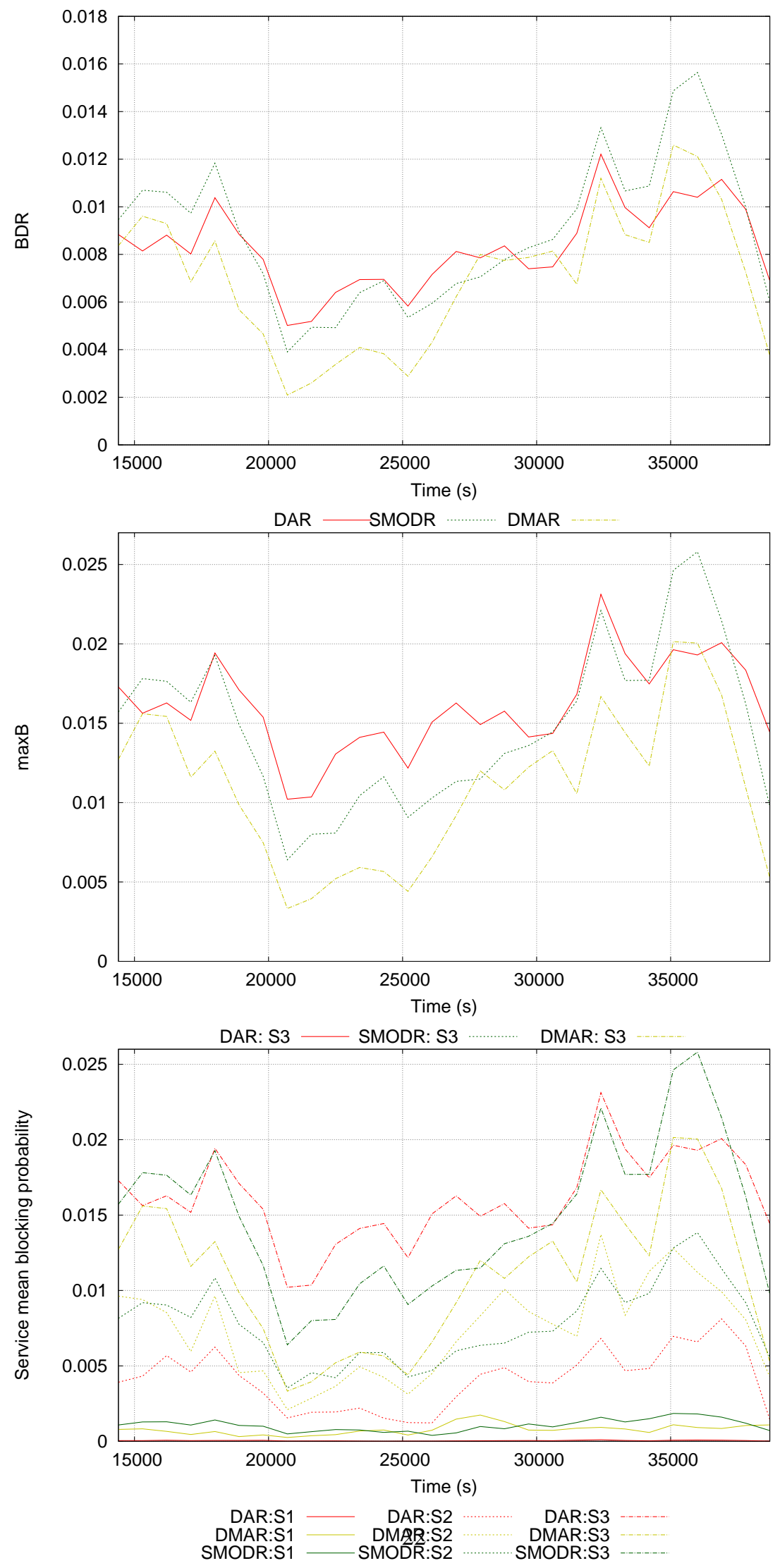

Figure 12: Network E - multi-service dynamic traffic. 
[8] Lin, H., Wang, S., Tsai, C., Hung, M.: Traffic Intensity Based Alternate Routing for All-Optical WDM Networks. J. Lightwave Technol. 26, 3604-3616 (2008)

[9] Lin, H., Wang, S., Hung, M.: Finding Routing Paths for Alternate Routing in All-Optical WDM Networks. J. Lightwave Technol. 26, 1432-1444 (2008)

[10] Kist, A., Harris, R.: Scheme for alternative packet overflow routing (SAPOR). In: Workshop on High Performance Switching and Routing, HPSR, pp. 269-274. IEEE (2003)

[11] Srivastava, S., Krithikaivasan, B., Beard, C., et al., D.M.: Benefits of Traffic Engineering using QoS Routing Schemes and Network Controls. Computer Communications 27, 387-399 (2004)

[12] Ash, G., McDysan, D.: Generic Connection Admission Control (GCAC) Algorithm Specification for IP/MPLS Networks. RFC 6601 (2012). URL http: //www.ietf.org/rfc/rfc6601.txt

[13] Martins, L., Francisco, C., Redol, J., Craveirinha, J., Clímaco, J., Monteiro, P.: Evaluation of a Multiobjective Alternative Routing Method in Carrier IP/MPLS Networks. In: Proceedings of the 8th International IFIP-TC 6 Networking Conference, NETWORKING 2009, pp. 195-206 (2009)

[14] Clímaco, J., Craveirinha, J., Girão-Silva, R.: Multicriteria analysis in telecommunication network planning and design: A survey. International Series in Operations Research and Management Science. 233, 1167-1233 (2016)

[15] Martins, L., Craveirinha, J., Clímaco, J., Gomes, T.: On a Bi-Dimensional Dynamic Alternative Routing Method. European Journal of Operational Research - Special Issue on Advances in Complex Systems Modeling 166(3), 828-842 (2005)

[16] Martins, L., Craveirinha, J., Clímaco, J.: A New Multiobjective Dynamic Routing Method for Multiservice Networks: Modelling and Performance. Computational Management Science 3(3), 225-244 (2006)

[17] Kelly, F.P.: Routing in circuit-switched networks: Optimization, shadow prices and decentralization. Advances in Applied Probability 20, 112-144 (1988)

[18] Francisco, C., Martins, L., Medhi, D.: Traffic model for Dynamic Multicriteria Alternative Routing for Single- and Multi-service ReservationOriented Networks. Tech. Rep. 1, INESC-Coimbra, available online: https://www.uc.pt/en/org/inescc/res_reports_docs/research_reports (2018)

[19] Kaufman, J.S.: Blocking in a shared resource environment. IEEE Transactions on Communications 29(10), 1474-1481 (1981)

[20] Mitra, D., A.Morrison, J.: Erlang capacity and uniform approximations for shared unbuffered resources. IEEE/ACM Transactions on Networking 2(6), 558-570 (1994)

[21] Medhi, D., Guptan, S.: Network dimensioning and performance of multiservice, multirate loss networks with dynamic routing. IEEE/ACM Transactions on Networking 5, 944-957 (1997) 
[22] Medhi, D.: QoS Routing Computation with Path Caching: A Framework and Network Performance. IEEE Communications Magazine 40(12), 106-113 (2002)

[23] Sivasankar, R., Ramam, S., Subramaniam, S., Rao, T., Medhi, D.: Some studies on the impact of dynamic traffic in a QoS-based dynamic routing environment. In: Proc. of 2000 IEEE International Conference on Communications (ICC), vol. 2, pp. 959-963 (2000)

[24] Mesquite Software: CSIM. http://www.mesquite.com/

[25] Chemouil, P., Filipiak, J., Gauthier, P.: Performance issues in the design of dynamically controlled circuit-switched networks. IEEE Communications Magazine 28(10), 90-95 (1990)

\section{A Traffic Matrices}

\section{A.1 Single service network}

Traffic "BH" in Table 1 is based on a voice service whose traffic pattern was made available by Sprint for the busiest hour for a 10-node network [21].

Table 1: Offered traffic matrices for the single service network.

\begin{tabular}{|c|c|c|c|c|c|c|c|c|c|c|c|}
\hline \multirow{2}{*}{ O-D } & \multirow{2}{*}{$\mathrm{BH}$} & \multicolumn{4}{|c|}{$7 \mathrm{BHs}$} & \multirow{2}{*}{ O-D } & \multirow{2}{*}{$\mathrm{BH}$} & \multicolumn{4}{|c|}{$7 \mathrm{BHs}$} \\
\hline & & Avg & $\mathrm{A}(\%)$ & $\mathrm{T}(\mathrm{h})$ & $\varphi\left(^{\circ}\right)$ & & & Avg & $\mathrm{A}(\%)$ & $\mathrm{T}(\mathrm{h})$ & $\varphi\left(^{\circ}\right)$ \\
\hline $1-2$ & 358 & 289 & 30 & 5 & 0 & $3-10$ & 750 & 649 & 21 & 4 & -112.5 \\
\hline $1-3$ & 1137 & 978 & 22 & 5 & 0 & $4-5$ & 1218 & 981 & 35 & 4 & -112.5 \\
\hline $1-4$ & 299 & 255 & 23 & 5 & 0 & $4-6$ & 921 & 775 & 25 & 4 & -112.5 \\
\hline $1-5$ & 338 & 284 & 25 & 5 & 0 & $4-7$ & 670 & 700 & 36 & 2 & -135 \\
\hline $1-6$ & 990 & 823 & 27 & 5 & 0 & $4-8$ & 323 & 282 & 22 & 4.5 & -50 \\
\hline $1-7$ & 456 & 490 & 26 & 2.5 & -234 & $4-9$ & 361 & 404 & 34 & 2 & -135 \\
\hline $1-8$ & 416 & 332 & 32 & 5 & 0 & $4-10$ & 372 & 315 & 24 & 4 & -112.5 \\
\hline $1-9$ & 238 & 254 & 32 & 2.5 & -234 & $5-6$ & 713 & 597 & 26 & 4 & -112.5 \\
\hline $1-10$ & 529 & 434 & 28 & 5 & 0 & $5-7$ & 557 & 680 & 14 & 2 & -135 \\
\hline $2-3$ & 766 & 649 & 24 & 5 & 0 & $5-8$ & 244 & 205 & 25 & 4 & -112.5 \\
\hline $2-4$ & 520 & 441 & 30 & 4 & -112.5 & $5-9$ & 302 & 374 & 15 & 2 & -135 \\
\hline $2-5$ & 378 & 311 & 28 & 5 & 0 & $5-10$ & 359 & 302 & 25 & 4 & -112.5 \\
\hline $2-6$ & 902 & 751 & 26 & 5 & 0 & $6-7$ & 2417 & 2819 & 10 & 3 & -300 \\
\hline $2-7$ & 399 & 413 & 33 & 2.5 & -234 & $6-8$ & 3375 & 2911 & 25 & 5 & 0 \\
\hline $2-8$ & 320 & 258 & 31 & 5 & 0 & $6-9$ & 1210 & 1439 & 11 & 2 & -135 \\
\hline $2-9$ & 227 & 233 & 35 & 2.5 & -234 & $6-10$ & 1679 & 1451 & 22 & 4 & -112.5 \\
\hline $2-10$ & 513 & 421 & 29 & 4 & -22.5 & $7-8$ & 661 & 677 & 34 & 2.5 & -234 \\
\hline $3-4$ & 1054 & 853 & 30 & 4 & -112.5 & $7-9$ & 2360 & 3714 & 33 & 4 & -292.5 \\
\hline $3-5$ & 1466 & 1174 & 32 & 4 & -112.5 & $7-10$ & 620 & 606 & 38 & 2.5 & -234 \\
\hline $3-6$ & 2215 & 1926 & 23 & 4 & -112.5 & $8-9$ & 467 & 576 & 15 & 2.5 & -234 \\
\hline $3-7$ & 1375 & 1403 & 37 & 2 & -135 & $8-10$ & 621 & 516 & 27 & 5 & 0 \\
\hline $3-8$ & 835 & 728 & 21 & 4 & -112.5 & $9-10$ & 407 & 497 & 14 & 2.5 & -234 \\
\hline $3-9$ & 745 & 770 & 35 & 2 & -135 & & & & & & \\
\hline
\end{tabular}

Dynamic traffic matrices present a time-varying traffic pattern that is mapped as a sinusoidal wave for each pair of nodes by considering the following time-dependent function: $\operatorname{Traf}(\mathrm{t})=\operatorname{Avg}(1+\operatorname{A} \sin (2 \pi f t+\varphi))$. The Sprint offered traffic matrices for the seven busiest hours period $([10,17[\mathrm{~h})$ inspired the mapping into the sinusoidal waves defined as "7 BHs" in Table 1. 


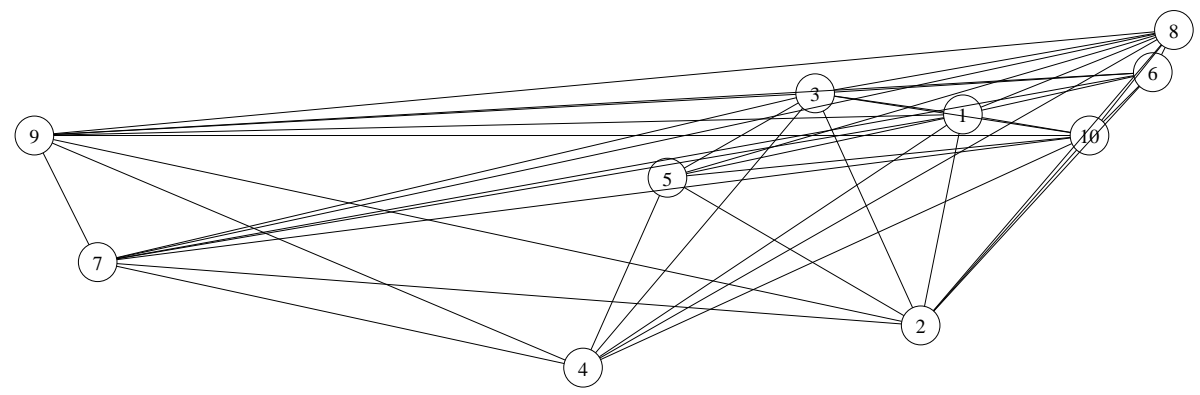

Figure 13: Network B - topology.

\section{A.2 Multi-service network}

Work in [11] applies to multi-service networks with a load distribution based on an actual service provider. This work was inspired by [11] leading to the following load distribution: $5\left(S_{1}\right): 20\left(S_{2}\right): 75\left(S_{3}\right)$. This study assumes that the base rate is the bandwidth in use by service $S_{1}$ and that all services require some multiple of this base rate. The multi-service networks were thus engineered with three services with the required bandwidth d=[1,6,10] for services $S_{1}, S_{2}$ and $S_{3}$, respectively.

Traffic for service $S_{1}$ is based on single service traffic. The traffic that is offered to services S2 and S3 in every situation is such that maintains the load distribution before mentioned. To reduce the simulation time, the average values used by each service are affected by a multiplicative factor $\mathrm{f}=[1 / 3,2 / 9,1 / 2]$ for services $\mathrm{S} 1, \mathrm{~S} 2$ and $\mathrm{S} 3$, respectively. In the dynamic traffic case, the amplitude variation is also affected by the same factor.

\section{B Test Networks}

Table 2 presents all the networks used in this study. The dimensioning of the single service networks was done in a simplistic manner based on the Sprint offered traffic matrices for the seven busiest hours period, assuming the use of direct routing and using the inverse of Erlang B formula as first approach for a reference blocking probability value.

Network A was adjusted by simulation based on the busiest hour traffic to get a mean network blocking probability of approximately $1 \%$ for DAR.

Network $\mathrm{C}$ was dimensioned based on the traffic for the three time instants corresponding to the peak values of the offered traffic in Figure 9 , and using the fixed point iterators in [18].

Network B is a sparser network and it resulted from the removal of approximately 10\% (5 links) of the links in Network A (see Figure 13). The first choice path for each of the pairs of nodes is fixed and the same regardless of the routing method. The capacities of the links that are used in the two-arcs first choice paths are increased, when compared with Network A, by the corresponding capacities of the removed links.

Multi-service networks D and E were obtained in a similar way as single service networks $\mathrm{A}$ and $\mathrm{C}$, respectively, and considering all the offered traffic as if it was single service. 
Table 2: Networks A, B, C, D, E: links capacities.

\begin{tabular}{c|c|c|c|c|c|c|c|c|c|c|c}
\hline O-D & $\mathrm{A}$ & $\mathrm{B}$ & $\mathrm{C}$ & $\mathrm{D}$ & $\mathrm{E}$ & $\mathrm{O}-\mathrm{D}$ & $\mathrm{A}$ & $\mathrm{B}$ & $\mathrm{C}$ & $\mathrm{D}$ & $\mathrm{E}$ \\
\hline $1-2$ & 363 & 363 & 140 & 2405 & 2211 & $3-10$ & 754 & 754 & 253 & 5015 & 4579 \\
$1-3$ & 1105 & 1105 & 415 & 7600 & 7131 & $4-5$ & 1108 & 1108 & 391 & 8410 & 6988 \\
$1-4$ & 325 & 1215 & 119 & 2012 & 1918 & $4-6$ & 890 & - & 302 & 6156 & 5475 \\
$1-5$ & 356 & 812 & 133 & 2272 & 2141 & $4-7$ & 809 & 809 & 311 & 6061 & 5192 \\
$1-6$ & 940 & 1830 & 364 & 6643 & 6099 & $4-8$ & 354 & 354 & 129 & 2196 & 2107 \\
$1-7$ & 583 & 583 & 214 & 3919 & 3602 & $4-9$ & 490 & 1020 & 184 & 3457 & 3020 \\
$1-8$ & 411 & 411 & 161 & 2792 & 2541 & $4-10$ & 392 & 392 & 131 & 2500 & 2267 \\
$1-9$ & 324 & 780 & 121 & 2145 & 1930 & $5-6$ & 699 & - & 237 & 4775 & 4241 \\
$1-10$ & 523 & 523 & 201 & 3546 & 3267 & $5-7$ & 788 & - & 267 & 4927 & 4813 \\
$2-3$ & 754 & 754 & 284 & 5123 & 4790 & $5-8$ & 268 & 1755 & 89 & 1645 & 1498 \\
$2-4$ & 530 & - & 181 & 3641 & 3168 & $5-9$ & 456 & - & 154 & 2741 & 2684 \\
$2-5$ & 388 & 388 & 147 & 2538 & 2358 & $5-10$ & 376 & 376 & 126 & 2411 & 2176 \\
$2-6$ & 863 & 863 & 332 & 6035 & 5564 & $6-7$ & 3025 & 3025 & 961 & 19614 & 18465 \\
$2-7$ & 499 & 499 & 190 & 3489 & 3098 & $6-8$ & 3121 & 3820 & 1222 & 23161 & 21190 \\
$2-8$ & 328 & 328 & 127 & 2164 & 1983 & $6-9$ & 1591 & 1591 & 538 & 10178 & 10017 \\
$2-9$ & 300 & 830 & 114 & 2019 & 1789 & $6-10$ & 1604 & 1604 & 545 & 11210 & 10120 \\
$2-10$ & 507 & 507 & 195 & 3457 & 3167 & $7-8$ & 784 & 1572 & 304 & 5756 & 5044 \\
$3-4$ & 973 & 973 & 337 & 7049 & 6055 & $7-9$ & 3948 & 3948 & 1095 & 31338 & 23915 \\
$3-5$ & 1313 & 1313 & 461 & 9861 & 8318 & $7-10$ & 708 & 708 & 281 & 5332 & 4576 \\
$3-6$ & 2099 & 2099 & 720 & 15048 & 13417 & $8-9$ & 676 & 676 & 232 & 4198 & 3906 \\
$3-7$ & 1553 & 1553 & 609 & 12223 & 10332 & $8-10$ & 612 & 612 & 233 & 4160 & 3853 \\
$3-8$ & 839 & 839 & 281 & 5585 & 5118 & $9-10$ & 591 & 591 & 201 & 3609 & 3554 \\
$3-9$ & 884 & 884 & 339 & 6624 & 5695 & & & & & & \\
\hline
\end{tabular}

